\title{
Silver Birch Pollen IgG4 Measurement
}

National Cancer Institute

\section{Source}

National Cancer Institute. Silver Birch Pollen Ig G4 Measurement. NCI Thesaurus. Code C130079.

A measurement of the silver birch pollen IgG4 in a biological specimen. 